\title{
Erratum: Some Cylindrical Symmetric Non-Static Perfect Fluid Distributions in General Relativity with Pressure Equal to Density ${ }^{1}$
}

\author{
S. R. Roy and S. Narain
}

(i) In equation (79) the value of $v_{4}$ should be zero and therefore $v_{1}^{2}=-A^{2}$. Hence the model in case (a.2.1) is unrealistic.

(ii) The right-hand side of equation (63) should read

$$
4\left[m(m-1)+\phi^{2} \frac{d}{d \phi}\left(\frac{1}{A} \frac{d A}{d \phi}\right)\right]
$$

and consequently the expressions for metrics (68) and (70), resepctively, should be changed to

$$
\begin{aligned}
d s^{2}= & \phi^{2 q} \exp (n \phi+l)\left(d T^{2}-d x^{2}\right) \\
& -\phi^{2 m} d y^{2}-\phi^{2(1-m)} d z^{2}
\end{aligned}
$$

and

$$
\begin{aligned}
d s^{2}= & \phi^{2 m(m-1)} \exp \left(h \phi^{2}+n \phi+\xi_{0}\right)\left(d T^{2}-d x^{2}\right) \\
& -\phi^{2 m} d y^{2}-\phi^{2(1-m)} d z^{2}
\end{aligned}
$$

where $q, n, l, h$, and $\xi_{0}$ are constants.

(iii) Expressions (80)-(82), respectively, should be changed as follows:

$$
8 \pi \rho=8 \pi p=\frac{1}{-U V} \frac{q-m(m-1)}{n^{2} A^{2} \phi^{2}}
$$

${ }^{1}$ This paper appeared in International Journal of Theoretical Physics, 20, 709 (1981). 


$$
\begin{aligned}
& v_{1}=\frac{-A x}{\left(T^{2}-x^{2}\right)^{1 / 2}} \\
& v_{4}=\frac{A T}{\left(T^{2}-x^{2}\right)}
\end{aligned}
$$

The reality conditions in this case are $T^{2}-x^{2}>0$ and $q-m(m-1)>0$.

(iv) Expressions (83)-(85), respectively, should be changed to

$$
\begin{aligned}
8 \pi \rho & =8 \pi p=\frac{4 h}{n^{2}\left(x^{2}-T^{2}\right)} \\
v_{1} & =\frac{-A T}{\left(x^{2}-T^{2}\right)^{1 / 2}} \\
v_{4} & =\frac{A x}{\left(x^{2}-T^{2}\right)}
\end{aligned}
$$

and the reality conditions for this case are $x^{2}-T^{2}>0$ and $h>0$. 